\title{
Effect of Monomer Functionality and Concentration on Gelation in Non-Linear Random Polymerization
}

\author{
Z. AHMED and R. F. T. STEPTo \\ Department of Chemistry, Quaid-i-Azam University, \\ Islamabad, Pakistan
}

(Received December 25, 1981)

\begin{abstract}
The effect of the functionality of a branch unit and its concentration on gelation during polyesterification were examined. The measured gel-points were compared with the values predicted by the Flory and Stockmayer theories of gelation. Considerable deviations from the theories were found for the system where equal reactivity of the functional groups was ensured.

KEY WORDS Non-Linear Random Polymerization / Monomer Functionality / Gel Points / Intramolecular Reaction /
\end{abstract}

Polymerization of monomers having a functionality greater than two leads to a network structure. The progressive formation of the network structure is reflected in a continual decrease in the soluble (sol) fraction and an increase in the insoluble (gel) fraction in the polymer system. At a certain point, the viscosity of the system increases infinitely and the reaction mixture is transformed from a liquid to a solid phase, usually called gel. This phenomenon known as gelation depends on various parameters, e.g., weight-average functionality, molecular weights of the monomers, and the reaction conditions (e.g., concentration of monomers and temperature).

The classical theories of Flory ${ }^{1}$ and Stockmayer ${ }^{2}$ provide the basic explanation for the occurrence of gelation in non-linear step-polymerization (network polymer). Experimentally, it has been found ${ }^{3,4}$ that gelation often occurs at a point more progressed in the reaction than predicted by Flory's theory. This discrepancy can be explained as a result of the unequal reactivities of similar functional groups, ${ }^{5}$ unequal concentrations of the reacting monomers used, and the formation of ring structures through intramolecular reaction. ${ }^{6-8}$ The general polymerization statistics including both unequal reactivity and intramolecular reactions have been formulated by Gordon and coworkers. ${ }^{9-11}$ The treatment so far developed for irreversible nonlinear polymerization is accurate only for the reactions have progressed to a small extent. ${ }^{12}$ The effect of weight-average functionality on gelation has been investigated by French and Strecker ${ }^{13}$ and Miller et al. ${ }^{14}$

According to Flory, ${ }^{1}$ the condition of gelation for a system comprising equimolar monomers of type $\mathrm{A}-<\mathrm{A}$ and $\mathrm{B}-\mathrm{B}$, where $\mathrm{A}$ can react only with $\mathrm{B}$, is given by

$$
\alpha_{c}=\frac{1}{(f-1)}
$$

where $f$ is the functionality of the branch unit and $\alpha$ is the probability that a polymer chain ends at a branch unit. At the critical gel-point, this probability should be equal to unity. Flory ${ }^{1}$ related $\left(\alpha_{c}\right)$ to $P_{\mathrm{A}}$ and $P_{\mathrm{B}}$, the extent to which the $\mathrm{A}$ and $\mathrm{B}$ monomer type reactions have proceeded, respectively, by

$$
\left(P_{\mathrm{A}} P_{\mathrm{B}}\right)_{\mathrm{c}}=\frac{1}{(f-1)}
$$

A more general relationship, however, has been given by Stockmayer and $\mathrm{Kahn}^{15}$ as

$$
\left(P_{\mathrm{A}} P_{\mathrm{B}}\right)_{\mathrm{c}}=\left(f_{\mathrm{A}}-1\right)^{-1}\left(f_{\mathrm{B}}-1\right)^{-1}
$$

where $f_{\mathrm{A}}$ and $f_{\mathrm{B}}$ are the weight-average functionalities of monomers A and B and represented by

$$
F_{\mathrm{A}}=\frac{\sum_{i} f_{\mathrm{A}-i}^{2} n_{\mathrm{A}-i}}{\sum_{i} f_{\mathrm{A}-i} n_{\mathrm{A}-i}}
$$




$$
F_{\mathrm{B}}=\frac{\sum_{i} f_{\mathrm{B}-\mathrm{i}}^{2} n_{\mathrm{B}-i}}{\sum_{i} f_{\mathrm{B}-i} n_{\mathrm{B}-i}}
$$

with $n$ being the number of moles of each species.

French and Strecker ${ }^{13}$ studied the effects of weight-average functionality on gelation, using (i) mixtures of stearic acid and sebacic acid in various ratios reacted with 1,1,1-propanetrimethane and (ii) mixtures of sebacic acid and 1,2,3-propanetricarboxylic acid in various ratios reacted with 1,4 butane diol. A comparison of the measured gel-points with those predicted by the theories of Flory (eq 1) and Stockmayer and Kahn (eq 2) showed a deviation of about $10 \%$ in the systems studied. Neglecting these deviations, French and Strecker concluded that the general equation of Stockmayer and Kahn predicts adequately the extent to which the reaction at the gel-point has proceeded.

In the present work, a gelation study was made on the polyesterification in which mixtures of a diol and a triol of different compositions (changing $f_{\mathrm{w}}$ from three to two) were allowed to react with sebacoyl chloride. The reactions were studied at $60^{\circ} \mathrm{C}$ with diglyme (diethylene glycol dimethyl ether) as the solvent.

\section{EXPERIMENTAL}

The polymeric alcohols (LHT 112, LHT 240, PPG 1025, PPG 2025) were supplied by Union Carbide Corporation. LHT 112 \& LHT 240 are the polypropylene oxide adducts of triols and PPG 1025 and PPG 2025 are the polypropylene oxide adducts of diols.

The choice of these polymeric alcohols was made on the basis of their narrow molecular weight distributions and functional groups separated by a sufficiently long chain, thus ensuring equal reactivity. The traces of water were removed by refluxing the polyols for $24 \mathrm{~h}$ period with four volumes of redistilled benzene. Water-benzene azeotropes were removed using 'Dean-Stark' apparatus. Benzene was removed by distillation under vacuum. The alcohols were left standing for $24 \mathrm{~h}$ at $0.1 \mathrm{mmHg}$ at $60^{\circ} \mathrm{C}$ to remove final traces of benzene. The alcohols so dried were analyzed by IR and by the more accurate Karl Fischer method. The water content of the alcohols was found to be less than $0.029 \%$ by wt.

Diglyme (solvent) supplied by BDH Ltd. was of SLR Grade. It was further purified by refluxing over sodium for four days until the sample withdrawn gave a negative test for hydroxyl groups and free water as judged by the absence of peaks at 2.8 and $3.0 u$ and $6.2 u$ in the IR spectrum. Sebacoyl chloride was supplied by BDH Ltd. and purified by vacuum distillation under inert atmosphere. The fraction which boiled in the temperature range $122-124^{\circ} \mathrm{C}$ at 0.08 to $0.09 \mathrm{mmHg}$ was collected.

The reaction was carried out in a flask at $60^{\circ} \pm 0.1^{\circ} \mathrm{C}$. Concentrations of hydroxyl and acid chloride groups were taken in equivalents. Acid chloride was allowed to react with hydroxyl groups to give an ester linkage and an equivalent amount of $\mathrm{HCl}$ in a non-reversible reaction. On quenching the sample withdrawn from the reaction flask with a mixture of methyl ethyl ketone/water/acetone, the unreacted acid chloride produced equivalent amounts of $\mathrm{HCl}$ and organic acid. As the reaction proceeded, the total acid concentration decreased by an amount equal to the ester linkage formed and was measured by titrating the mixture against standard alkali. The concentration of $\mathrm{Cl}$ ions was measured by titrating against a standard silver nitrate solution. The difference between the two values gave the concentration of organic acid, and hence the extent of reaction was exactly determined.

The extent of reaction at the gel-point was determined by extrapolation of the conversion curve to the gel-point. Gelation was defined as the point at which there was a sudden fall in the fluidity of the reaction mixture followed by twisting of the polymer mass about the mechanical stirrer used to homogenize the reaction mixture.

\section{SUBSIDIARY KINETIC EXPERIMENTS}

In order to establish the effects of the weightaverage functionality of branch units on the gelpoint, kinetic experiments were performed to choose a diol which could be combined with a triol. Mixtures of a diol and a triol in different proportions were prepared and the weight-average functionality of the triol was systematically reduced from 3 to 2 by increasing the amount of diol. It was necessary for the hydroxyl groups of diol and triol to have equal reactivities towards acid chloride group so as to allow for a more certain interpretation of the results.

The kinetic data for the reaction between these 
Table I. Second-order rate constants for reaction between polymeric alcohols and sebacoyl chloride in diglyme at $60^{\circ} \mathrm{C}$

\begin{tabular}{cccc}
\hline Polyol & Mol [OH] $/ \mathrm{kg}^{-1}$ & Mol $[\mathrm{COCl}] / \mathrm{kg}^{-1}$ & $k \times 10^{-3} / \mathrm{kg} \mathrm{mol}^{-1} \mathrm{~s}^{-1}$ \\
\hline LHT 112 & 1.0542 & 0.9823 & 1.207 \\
PPG 1025 & 1.0278 & 1.0005 & 1.153 \\
LHT 240 & 1.0009 & 0.9876 & 1.068 \\
\hline
\end{tabular}

polyols and sebacoyl chloride showed that PPG 1025 has a reactivity very close to that of LHT 112 . The reactions were found to follow a second-order kinetics. The values of the second-order rate con-<smiles>CC(O)COCC1CCOCC(C)COCC(C)CO1</smiles>

LHT 112 (polypropylene oxide adducts of 1,2,6 hexanetriol)

Here $\bar{n}$ is the number-average degree of polymerization of each branch. The number-average molecular weights of LHT 112 and PPG 1025 were $1478 \pm 3$ and $1034 \pm 3$, respectively. Different proportions of these polyols were allowed to react with sebacoyl chloride and the extent of reaction at gelpoint was determined.

\section{RESULTS AND DISCUSSION}

Experimental data are given in Table II, where $\rho$ represents the number of hydroxyl groups belonging to the branch unit relative to the total number of hydroxyl groups present in the mixture and $r$ expresses the ratio $(\mathrm{COCl}) /(\mathrm{OH})$. The gelation data are given in Table III, where $f_{\mathrm{N}}$ and $f_{\mathrm{w}}$ are the number-average and weight-average functionalities of the polymeric alcohols used.

Experimental data obtained may be interpreted on the basis of the theories of gelation of Flory, ${ }^{1}$ Stockmayer, ${ }^{2}$ and Kahn. ${ }^{15}$ In Table III, the ratio of observed to predicted gel-points versus the functionality of polyol is given. The deviation in the stants are given in Table I.

On the basis of the kinetic experiments LHT 112 and PPG 1025 were chosen. The structural formulae of these polymeric alcohols are<smiles>CC(O)COCCOCC(C)O</smiles>

PPG 1025 (polypropylene oxide adducts of ethyleneglycol)

predicted extent of reaction at the gel-point ranges up to $10-15 \%$. The observed deviation shows a systematic trend with the extent of the reaction at the gel-point, increasing as the reaction mixture is diluted. The experimental systems $(1.1-1.6)$ studied at high initial concentrations show a minimum deviation, and the results may be considered to agree with the values predicted by the theories of gelation. ${ }^{1,2}$ However, the gel-points obtained for experimental systems $(3.1-3.6)$ using a low concentration show large deviation which cannot be ignored. It was found that the deviation increases systematically with dilution and becomes maximum at infinite dilution.

Figure 1 shows a comparison of the observed extent of the reaction at the gel-point with that predicted. A systematic shifts from the predicted extent of the reaction at the gel-point is evident. As the functionality of polyol decreases, the gel-point is observed at a greater reaction extent. Also as the system becomes more dilute observed extent of reaction deviates more from the theory.

Similar deviations were also observed by French 
Table II. Initial concentrations and ratios of reactants System 1-3: LHT $112+$ PPG 1025 + sebacoyl chloride in diglyme $60^{\circ} \mathrm{C}$.

\begin{tabular}{|c|c|c|c|c|c|c|}
\hline \multirow{2}{*}{$\begin{array}{c}\text { Exptl. } \\
\text { No. }\end{array}$} & \multirow{2}{*}{$\frac{\text { LHT } 112}{\mathrm{~mol}[\mathrm{OH}] \mathrm{kg}^{-1}}$} & \multirow{2}{*}{$\frac{\text { PPG } 1025}{\mathrm{~mol}[\mathrm{OH}] \mathrm{kg}^{-1}}$} & \multirow{2}{*}{$\frac{\begin{array}{c}\text { Sebacoyl } \\
\text { chloride }\end{array}}{\mathrm{mol}[\mathrm{COCl}] \mathrm{kg}^{-1}}$} & \multirow{2}{*}{$\frac{\begin{array}{c}\text { Reciprocal } \\
\text { concentration }\end{array}}{\mathrm{kg} \mathrm{mol}^{-1}}$} & \multicolumn{2}{|c|}{ On weight basis } \\
\hline & & & & & $\rho$ & $r$ \\
\hline 1.1 & 1.5005 & - & 1.5013 & 0.3331 & 1.0000 & 1.0005 \\
\hline 1.2 & 1.2142 & 0.2862 & 1.4761 & 0.3360 & 0.8092 & 0.9838 \\
\hline 1.3 & 0.9746 & 0.5300 & 1.4908 & 0.3338 & 0.6477 & 0.9908 \\
\hline 1.4 & 0.7496 & 0.7556 & 1.4958 & 0.3332 & 0.4980 & 0.9937 \\
\hline 1.5 & 0.5163 & 0.9818 & 1.4086 & 0.3291 & 0.3352 & 1.0003 \\
\hline 1.6 & 0.2885 & 1.2122 & 1.5060 & 0.3326 & 0.1923 & 1.0035 \\
\hline 2.1 & 0.8957 & - & 0.9311 & 0.5374 & 1.0000 & 1.0395 \\
\hline 2.2 & 0.7292 & 0.1734 & 0.8923 & 0.5571 & 0.8079 & 0.9886 \\
\hline 2.3 & 0.5864 & 0.3099 & 0.9318 & 0.5470 & 0.6542 & 0.0396 \\
\hline .2 .4 & 0.4494 & 0.4493 & 0.9197 & 0.5505 & 0.5000 & 0.0214 \\
\hline 2.5 & 0.3122 & 0.5881 & 0.8898 & 0.5586 & 0.3468 & 0.9883 \\
\hline 2.6 & - & 0.9002 & 0.8724 & 0.5641 & 0.0000 & 0.9691 \\
\hline 3.1 & 0.6526 & - & 0.6542 & 0.7652 & 1.0000 & 0.0024 \\
\hline 3.2 & 0.5274 & 0.1244 & 0.6453 & 0.7709 & 0.8091 & 0.9899 \\
\hline 3.3 & 0.4286 & 0.2249 & 0.6496 & 0.7674 & 0.6558 & 0.9940 \\
\hline 3.4 & 0.3246 & 0.3256 & 0.6640 & 0.7609 & 0.4992 & 0.0212 \\
\hline 3.5 & - & 0.6515 & 0.6366 & 0.7763 & 0.0000 & 0.9771 \\
\hline
\end{tabular}

Table III. Gelation data

System 1-3: LHT $112+$ PPG $1025+$ sebacoyl chloride in diglyme at $60^{\circ} \mathrm{C}$.

\begin{tabular}{|c|c|c|c|c|c|c|c|}
\hline \multirow{2}{*}{$\begin{array}{l}\text { Exptl. } \\
\text { No. }\end{array}$} & \multirow{2}{*}{$F_{\mathrm{N}}$} & \multirow{2}{*}{$f_{\mathrm{W}}$} & \multirow{2}{*}{$\begin{array}{c}\text { Calculated } / \% \\
\text { gel-point }\end{array}$} & \multicolumn{2}{|c|}{ Observed $\%$ gel-point } & \multirow{2}{*}{ Obsd/pred. } & \multirow{2}{*}{$\left(P_{\mathrm{A}} P_{\mathrm{B}}\right)_{\mathrm{c}}^{-}$} \\
\hline & & & & $P_{\mathrm{A}}$ & $P_{\mathrm{B}}$ & & \\
\hline 1.1 & 2.988 & 2.988 & 70.7 & 73.5 & 73.4 & 1.04 & 1.8516 \\
\hline 1.2 & 2.81 & 2.86 & 72.6 & 77.9 & 79.2 & 1.07 & 1.6179 \\
\hline 1.3 & 2.65 & 2.73 & 74.1 & 81.9 & 82.6 & 1.05 & 1.4778 \\
\hline 1.4 & 2.50 & 2.60 & 76.7 & 87.4 & 87.9 & 1.14 & 1.3021 \\
\hline 1.5 & 2.34 & 2.44 & 80.5 & 90.9 & 90.8 & 1.13 & 1.2120 \\
\hline 1.6 & 2.19 & 2.26 & 86.3 & 94.7 & 94.3 & 1.10 & 1.1193 \\
\hline 2.1 & 2.988 & 2.988 & 72.1 & 74.9 & 77.9 & 1.04 & 1.7120 \\
\hline 2.2 & 2.81 & 2.86 & 72.5 & 80.3 & 81.2 & 1.11 & 1.5323 \\
\hline 2.3 & 2.65 & 2.73 & 77.8 & 85.9 & 82.6 & 1.11 & 1.4087 \\
\hline 2.4 & 2.50 & 2.60 & 79.9 & 90.4 & 88.5 & 1.13 & 1.2511 \\
\hline 2.5 & 2.34 & 2.44 & 82.8 & 94.9 & 95.9 & 1.15 & 1.0984 \\
\hline 2.6 & 2.00 & 2.00 & 98.4 & - & - & - & - \\
\hline 3.1 & 2.998 & 2.988 & 70.8 & 79.2 & 79.0 & 1.12 & 1.5972 \\
\hline 3.2 & 2.81 & 2.86 & 72.9 & 82.2 & 83.1 & 1.13 & 1.4643 \\
\hline 3.3 & 2.65 & 2.73 & 75.6 & 85.4 & 85.9 & 1.13 & 1.3635 \\
\hline 3.4 & 2.50 & 2.60 & 79.9 & 92.3 & 90.4 & 1.15 & 1.1994 \\
\hline 3.5 & 2.00 & 2.00 & 98.8 & - & - & - & - \\
\hline
\end{tabular}

and Strecker ${ }^{13}$ who, however, ignored the deviations and concluded that the theories of gelation $^{1,2}$ are adequate for predicting gel-points. Also, for the experimental systems they studied, ${ }^{13}$ the reactivity of the functional groups of mono-, di-, or tri-basic acid towards alcohols was not assured to be equal. For a more reliable interpretation of the results, a kinetic study on the reaction of different 


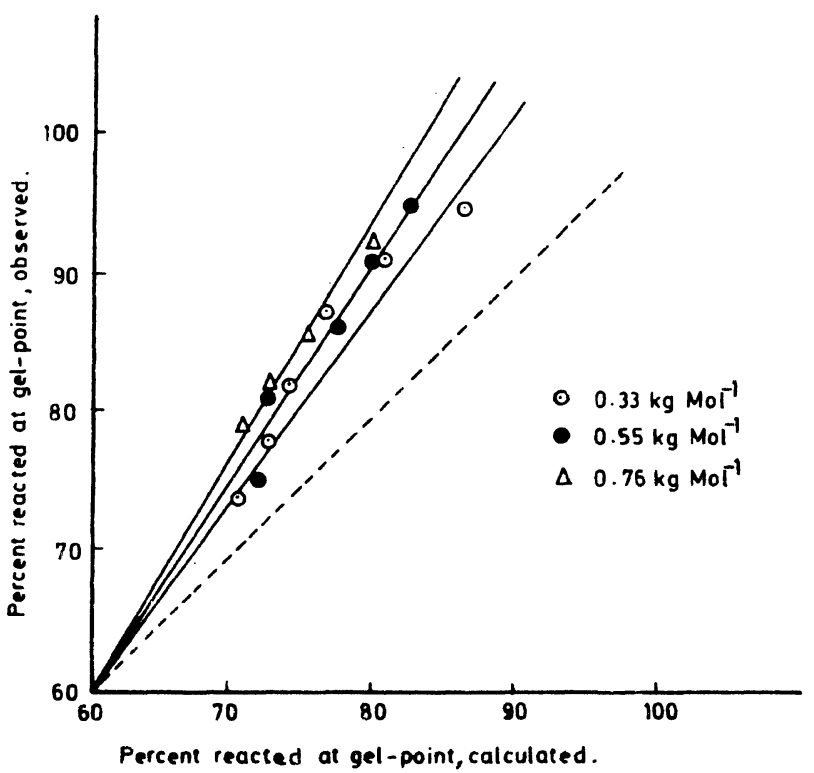

Figure 1. Deviation from predicted gel-point $v s$. functionality base moles.

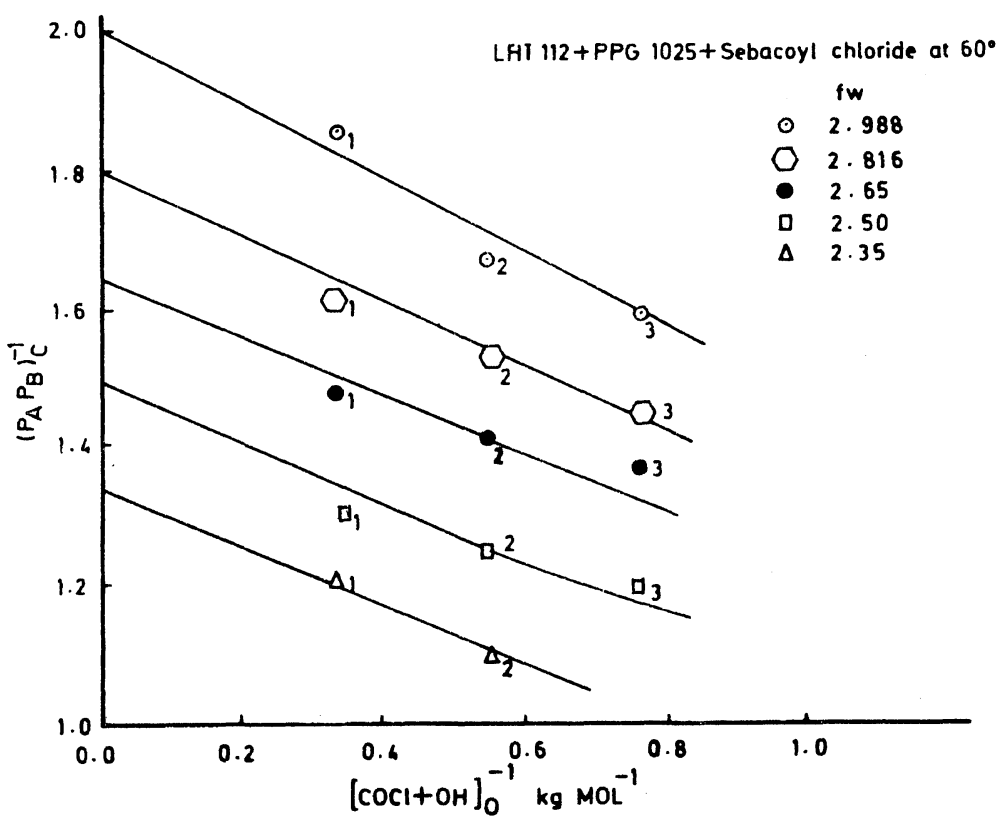

Figure 2. $\left(P_{\mathrm{A}} P_{\mathrm{B}}\right)_{\mathrm{c}}^{-1} v s$. initial dilution.

polyols with sebacoyl chloride was made in the present work. A suitable combination of a triol (LHT 112) and a diol (PPG 1025) having approx- imately the same value of rate constants (Table I) was chosen. For the present experimental system eq 3 can be rearranged as 


$$
\left(P_{\mathrm{A}} P_{\mathrm{B}}\right)_{\mathrm{c}}{ }^{-1}=\left(f_{\mathrm{w}}-1\right)
$$

where $f_{\mathrm{w}}$ is the weight-average functionality of polyol and ranges from 3 to 2.26 .

In Figure $2,\left(P_{\mathrm{A}} P_{\mathrm{B}}\right)^{-1}$ is plotted against the initial dilution. By extrapolation of these curves to infinite concentration, limiting values of $\left(P_{\mathrm{A}} P_{\mathrm{B}}\right)_{\mathrm{c}}^{-1}$ equal to $\left(f_{\mathrm{w}}-1\right)$ are obtained. The theories of Flory, Stockmayer, and Kahn thus predict gel-points adequately only at infinite concentration. The observed value of $\left(P_{\mathrm{A}} P_{\mathrm{B}}\right)_{\mathrm{c}}^{-1}$, however, is always lower and decreases as the dilution of the reaction system increases. A factor which is possibly of major significance in the deviation is the intramolecular reaction. This reaction as it competes with the intermolecular reactions does not contribute to the growth of the polymer network and thus retards gelation. The above-mentioned theories of gelation fail to take into consideration the contribution of intramolecular reaction and consequently do not predict gel-points for dilute systems, in which the intramolecular reaction must be taken into account.

\section{REFERENCES}

1. P. J. Flory, J. Phys. Chem., 46, 132 (1942).

2. W. H. Stockmayer, J. Chem. Phys., 12, 125 (1944).

3. F. P. Price, J. H. Gibbs, and B. H. Zimm, J. Phys. Chem., 62, 972 (1958).

4. R. F. T. Stepto, Faraday Discuss Chem., 57, 69 (1974).

5. W. H. Stockmayer, L. L. Weil, "Advancing Fronts in Chemistry," Reinhold, New York, 1945, Chapter IV.

6. R. W. Kilb, J. Phys. Chem., 62, 969 (1958).

7. H. L. Frisch, Paper presented to The American Chemical Society, 128th Meeting Polymer Div., Minneapolis, 1955.

8. J. L. Stanford and R. F. T. Stepto, J. Chem. Soc., Faraday Trans. 1 (1975).

9. M. Gordon, Proc. R. Soc. London, Ser. A, 268, 240 (1962).

10. M. Gordon and W. B. Temple, Makromol. Chem., 160, 263 (1972).

11. M. Gordon and G. R. Scantlebury, Proc. R. Soc. London, Ser. A, 292, 380 (1966).

12. W. B. Temple, Macromol. Chem., 160, 277 (1972).

13. D. M. French and R. A. H. Strecker, J. Macromol. Sci. Chem., A5(S), 893 (1971).

14. D. R. Miller, E. M. Valles, and C. W. Macosko, Polym. Eng. Sci., 19, 272 (1979).

15. A. Kahn, J. Polym. Sci., 9, 69 (1952); ibid., 11, 424 (1953). 